\title{
UvA $\underset{x}{\underset{x}{x}}$ ECONOMETRICS
}

Discussion Paper: 2013/04

Tail probabilities and partial moments for quadratic forms in multivariate generalized hyperbolic random vectors

\author{
S.A. Broda
}

www.ase.uva.nl/uva-econometrics

\section{Amsterdam School of Economics}

Department of Economics \& Econometrics

Valckenierstraat 65-67

1018 XE AMSTERDAM

The Netherlands

UvA $\underset{\substack{\mathbf{x} \\ \mathbf{x}}}{\stackrel{\boldsymbol{x}}{\mathbf{x}}}$ UNIVERSITEIT VAN AMSTERDAM

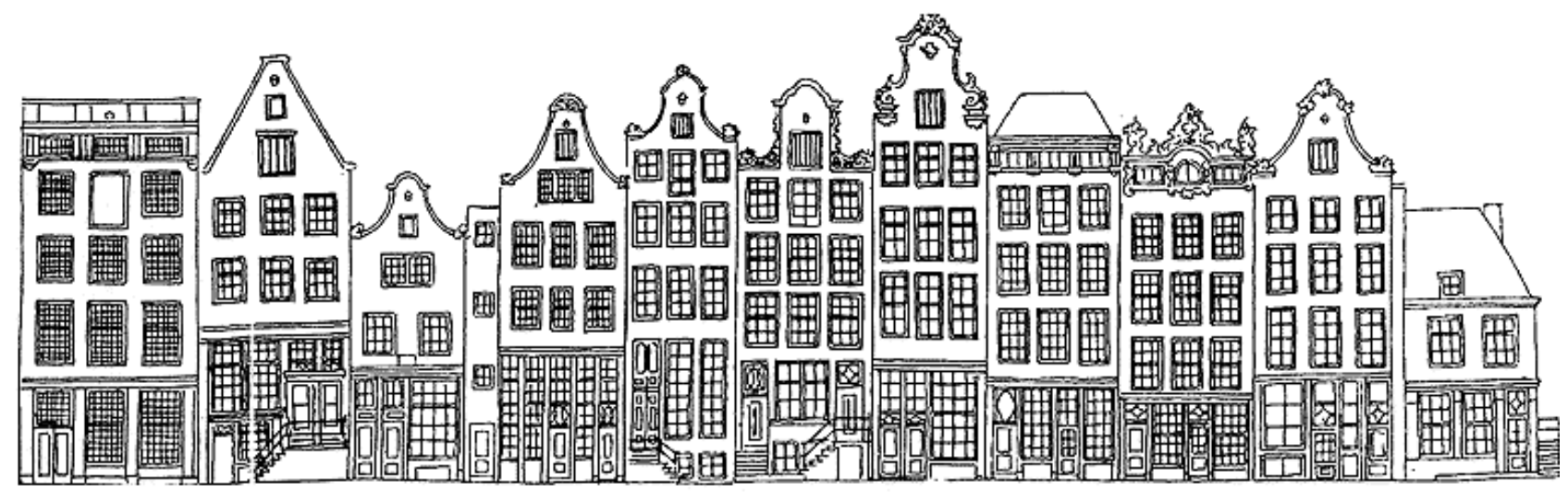




\title{
Tail Probabilities and Partial Moments for Quadratic Forms in Multivariate Generalized Hyperbolic Random Vectors
}

\author{
Simon A. Broda ${ }^{\dagger \ddagger *}$ \\ $\dagger$ Department of Economics and Econometrics, University of Amsterdam \\ $\ddagger$ Tinbergen Institute Amsterdam
}

May 2013

\begin{abstract}
Countless test statistics can be written as quadratic forms in certain random vectors, or ratios thereof. Consequently, their distribution has received considerable attention in the literature. Except for a few special cases, no closed-form expression for the cdf exists, and one resorts to numerical methods. Traditionally the problem is analyzed under the assumption of joint Gaussianity; the algorithm that is usually employed is that of Imhof (1961). The present manuscript generalizes this result to the case of multivariate generalized hyperbolic (MGHyp) random vectors. The MGHyp is a very flexible distribution which nests, among others, the multivariate $t$, Laplace, and variance gamma distributions. An expression for the first partial moment is also obtained, which plays a vital role in financial risk management. The proof involves a generalization of the classic inversion formula due to Gil-Pelaez (1951). Two applications are considered: first, the finite-sample distribution of the 2SLS estimator of a structural parameter. Second, the Value at Risk and Expected Shortfall of a quadratic portfolio with heavy-tailed risk factors.
\end{abstract}

Key Words: Finite Samples; Characteristic Function; Transform Inversion; 2SLS; CVaR; Expected Shortfall

JEL Classification: C16, C36, C63, G11, G32.

Mathematics Subject Classification (2010): 62E15, 62J05, 62P05, 65R32, 91G10, 91G60, 91G70

*E-mail address: s.a.broda@uva.nl 


\section{Introduction}

The generalized hyperbolic (GHyp) distribution was introduced by Barndorff-Nielsen (1977) in the context of describing the log size of particles, but has since found applications in a variety of fields, notably finance: an early reference in the latter field is Eberlein and Keller (1995). Its multivariate extension, to be referred to as the MGHyp in the sequel, has first been discussed in Blæsild and Jensen (1981). It, too, has received considerable attention in the finance literature. The reason for the popularity of the (M)Ghyp is its flexibility: it can model thin, heavy, and semi-heavy tails, and nests many popular distributions as special cases, such as the Student's t, Laplace, Variance Gamma, Hyperbolic, and Normal Inverse Gaussian distributions and their multivariate extensions, all of which have been applied successfully in financial modelling, see Hellmich and Kassberger (2011) and the references therein.

The present manuscript is concerned with quadratic forms in MGHyp random vectors, or more precisely, the sum of a quadratic and a linear form. Quadratic forms in random variables arise in a variety of applications; in Econometrics, many testing problems in linear models lead to statistics whose null distribution can be expressed as that of a quadratic form. Some well-known examples are Durbin and Watson's (1950) test for autocorrelation, Dickey and Fuller's (1979) test for a unit root (in its "coefficient" form), and the KPSS test for stationarity (Kwiatkowski et al., 1992). Consequently, a number of authors have considered algorithms for evaluating the distribution function of such random variables (see, e.g., the references in Forchini, 2002, Section 2.2). Typically, these algorithms involve inverting the characteristic function of the random variable of interest via the inversion formula of Gil-Pelaez (1951). Among them, Imhof's (1961) result appears to be the most widely used.

Unfortunately, the only case in which the characteristic function of a quadratic form is tractable is that in which the random vector entering it is Gaussian. In many applications, this assumption is too restrictive. The only paper of which the author is aware that dispenses with this assumption is that of Glasserman et al. (2002). In it, the authors show how to evaluate the distribution function of a quadratic form in a multivariate $t$ random vector. Their device is to express the probability of interest in terms of a certain auxiliary random variable, which, unlike the quadratic form of interest, possesses a tractable characteristic function. The first contribution of the present paper is the generalization of their result to the entire class of MGhyp distributions.

As an application, we consider the distribution of the two stage least squares (2SLS) estimator of a structural parameter in a simultaneous system of equations. The distribution of the 2SLS estimator under normality has been studied intensively; see Richardson (1968), Sawa (1969), Anderson and Sawa (1973), and Holly and Phillips (1979). In the wake of weak instrument asymptotics, it has been realized that the finite sample distribution is of much broader use than was originally recognized, thus spurring renewed interest in the topic, as evidenced by the work of Nelson and Startz (1990a,b), Maddala and Jeong (1992), and Woglom (2001), and the papers by Hillier (2006), Forchini (2006) and Phillips (2006), which comprise the entire miscellanea section of that journal issue. To the best of the author's knowledge, only Knight (1986) has considered the distribution under non-Gaussianity, specifically when the error distribution is expandable 
in an Edgeworth-type expansion. Here, we express the estimator as a linear plus a quadratic form in the innovation vector, as in Cribbett et al. (1989). The results of the present paper then facilitate the computation of the exact sampling distribution under MGhyp innovations, and with an arbitrary but known covariance structure.

The second contribution of the paper concerns partial moments. Consider a random variable $X$ with finite first moment. The quantity

$$
\mathbb{E}[X \mid X \leq x]
$$

that is, the expectation of $X$, conditional on falling in its own tail, has received considerable interest in the literature recently. In risk management, if $X$ denotes the return on a financial position whose distribution is continuous at its $q$ th quantile $x_{q},-\mathbb{E}\left[X \mid X \leq x_{q}\right]$ is known as the expected shortfall, conditional value at risk (CVaR), or tail conditional expectation, depending on author and context. The relevance of this quantity derives from the fact that unlike the more widely used Value at Risk (VaR), it defines a coherent risk measure in the sense of Artzner et al. (1999). The random variable of interest is often characterized most conveniently in terms of its characteristic function, possibly because its distribution arises from a convolution, as occurs in forming portfolios. Therefore, it is desirable to express the tail conditional expectation in terms of the characteristic function directly. As such, a number of authors have obtained expressions that facilitate such a computation; examples are Martin (2006), Kim et al. (2009), Broda and Paolella (2009), Pinelis (2010), and Bormetti et al. (2010). The representation of the moment generating function of a truncated random variable given in Butler and Wood (2004) can also be used for this purpose.

The aforementioned results all require, however, that the characteristic function be analytic in a strip containing the real axis, implying the existence of a moment generating function and hence all moments. They therefore fail for the heavy-tailed distributions that are ubiquitous in finance. The second contribution of the present paper is to provide an expression that is valid without such a restriction; in fact, in Section 2.1 below, a more general result will be proven which expresses the $n$th partial moment of $X$, provided it exists, in terms of the characteristic function. The result is a direct generalization of the inversion formula for the distribution function derived in Gil-Pelaez (1951), to which it collapses for $n=0$.

In Section 2.2, the result is generalized to ratios of random variables. This is the third contribution of the paper, and is of interest because numerous important distributions, such as the Student's $t$, permit a stochastic representation of this form. In particular, it allows us to derive an expression for the partial expectation of a quadratic form in a MGhyp random vector in Section 3, thus generalizing the results of Yueh and Wong (2010) and Broda (2011), which deal with the Gaussian and multivariate $t$ cases, respectively. As an application, we consider the expected shortfall of a quadratic portfolio, as arises from a delta-gamma approximation.

The structure of the paper is as follows. Section 2 derives general inversion formulae for partial moments. Section 3 provides computable expressions for the tail probabilities and partial expectations of a linear plus a quadratic form in a MGhyp random vector. Section 4 details two 
applications: the distribution function of the 2SLS estimator, and the Value at Risk and Expected Shortfall of a quadratic portfolio. Section 5 concludes.

\section{Inversion Formulae for Partial Moments}

\subsection{General Case}

Let $F(x)$ denote the distribution function of $X$. For $n \in\{0,1,2, \ldots\}$, define $G_{n}(x) \equiv \int_{-\infty}^{x} x^{n} d F(x)$, so that $F(x) \equiv G_{0}(x)$, and observe that at every point of continuity of $F$ (and hence $G_{n}$ ),

$$
\mathbb{E}\left[X^{n} \mid X \leq x\right]=\frac{G_{n}(x)}{F(x)} .
$$

The following result will be proven.

Theorem 2.1 Inversion Formula for Partial Moments. If the nth moment of $X, n \in$ $\{0,1,2, \ldots\}$, is finite and $F(x)$ is continuous at $x$, then

$$
G_{n}(x)=\frac{\varphi^{(n)}(0)}{2 \mathrm{i}^{n}}-\frac{1}{\pi} \int_{0}^{\infty} \operatorname{Im}\left[\frac{\mathrm{e}^{-\mathrm{i} t x} \varphi^{(n)}(t)}{\mathrm{i}^{n} t}\right] \mathrm{d} t,
$$

where $\varphi^{(n)}(t)$ is the $n$th derivative of the characteristic function of $X$.

Proof. Denote by $\varphi(t)$ the characteristic function of $X$. If the $n$th moment of $X$ is finite, then from Corollary 2 to Theorem 2.3.1 of Lukacs (1970),

$$
\varphi^{(n)}(t)=\mathrm{i}^{n} \int_{-\infty}^{\infty} x^{n} e^{\mathrm{i} t x} \mathrm{~d} F(x) .
$$

As in Gil-Pelaez (1951), define

$$
\operatorname{sign}(y-x)=\frac{2}{\pi} \int_{0}^{\infty} \frac{\sin t(y-x)}{t} \mathrm{~d} t=\left\{\begin{array}{rc}
-1, & y<x \\
0, & y=x \\
1, & y>x
\end{array}\right.
$$

and observe that

$$
\begin{aligned}
\int_{-\infty}^{\infty} \operatorname{sign}(y-x) y^{n} \mathrm{~d} F(y) & =\int_{x}^{\infty} y^{n} \mathrm{~d} F(y)-\int_{-\infty}^{x} y^{n} \mathrm{~d} F(y) \\
& =\left[\mathbb{E}\left[X^{n}\right]-G_{n}(x)\right]-G_{n}(x)=\frac{\varphi^{(n)}(0)}{\mathrm{i}^{n}}-2 G_{n}(x) .
\end{aligned}
$$


Then for $0<\epsilon<T$,

$$
\begin{aligned}
\frac{1}{\pi} \int_{\epsilon}^{T} \frac{\mathrm{e}^{-\mathrm{i} t x} \varphi^{(n)}(t)-\mathrm{e}^{\mathrm{i} t x} \varphi^{(n)}(-t)}{\mathrm{i}(t+1} t & =\frac{1}{\pi} \int_{\epsilon}^{T} \int_{-\infty}^{\infty} \frac{\mathrm{e}^{\mathrm{i} t(y-x)}-\mathrm{e}^{-\mathrm{i} t(y-x)}}{\mathrm{i} t} y^{n} \mathrm{~d} F(y) \mathrm{d} t \\
& =\frac{2}{\pi} \int_{\epsilon}^{T} \int_{-\infty}^{\infty} \frac{\sin (t(y-x))}{t} y^{n} \mathrm{~d} F(y) \mathrm{d} t \\
& =\frac{2}{\pi} \int_{-\infty}^{\infty} \int_{\epsilon}^{T} \frac{\sin (t(y-x))}{t} \mathrm{~d} t y^{n} \mathrm{~d} F(y),
\end{aligned}
$$

where the exchange of the order of integration is permissible because for each fixed value of $y$,

$$
\left|\frac{\sin t(y-x)}{t}\right|<\frac{1}{\epsilon}
$$

It remains to take the limit for $\epsilon \rightarrow 0$ and $T \rightarrow \infty$. Because the integral in $t$ is a continuous function of $\epsilon$ and $T$ with a bounded modulus, it is possible to pass the limit through the integral sign, to find

$$
\begin{aligned}
\lim _{\substack{\epsilon \rightarrow 0 \\
T \rightarrow \infty}} \frac{1}{\pi} \int_{\epsilon}^{T} \frac{\mathrm{e}^{-\mathrm{i} t x} \varphi^{(n)}(t)-\mathrm{e}^{\mathrm{i} t x} \varphi^{(n)}(-t)}{\mathrm{i}^{n+1} t} \mathrm{~d} t & =\frac{2}{\pi} \int_{-\infty}^{\infty} \lim _{\substack{\epsilon \rightarrow 0 \\
T \rightarrow \infty}} \int_{\epsilon}^{T} \frac{\sin (t(y-x))}{t} \mathrm{~d} t y^{n} \mathrm{~d} F(y) \\
& =\int_{-\infty}^{\infty} \operatorname{sign}(y-x) y^{n} \mathrm{~d} F(y)=\frac{\varphi^{(n)}(0)}{\mathrm{i}^{n}}-2 G_{n}(x) .
\end{aligned}
$$

Thus

$$
G_{n}(x)=\frac{\varphi^{(n)}(0)}{2 \mathrm{i}^{n}}-\frac{1}{2 \pi} \int_{0}^{\infty} \frac{\mathrm{e}^{-\mathrm{i} t x} \varphi^{(n)}(t)-\mathrm{e}^{\mathrm{i} t x} \varphi^{(n)}(-t)}{\mathrm{i}^{n+1} t} \mathrm{~d} t
$$

The result follows upon noting that $\varphi^{(n)}(t) / \mathrm{i}^{n}$ is the complex conjugate of $\varphi^{(n)}(-t) / \mathrm{i}^{n}$.

A few remarks are in order. First, the integral in the theorem does not, in general, converge absolutely, and must be interpreted as an improper Riemann integral. This is similar to the inversion integral of Gil-Pelaez (1951) for the distribution function, as remarked by Wendel (1961). It may be shown however that the integral converges absolutely under the Rosén-type condition (Rosén, 1961)

$$
\int_{-\infty}^{\infty} \log (1+|x|)|x|^{n} \mathrm{~d} F(x)<\infty
$$

Finally, Theorem 2.1 will clearly be most useful in situations where the integral permits no analytical solution, and must be evaluated by means of numerical quadrature schemes. In such cases, the doubly exponential transformation of Ooura and Mori (1991) may benefit the numerics.

\subsection{Ratios of Random Variables}

Consider a bivariate random variable $\left(X_{1}, X_{2}\right)$ and let $R \equiv X_{1} / X_{2}$. The following theorem will be proven.

Theorem 2.2 Partial Expectation of A Ratio. If (i) the characteristic function $\varphi_{X_{1}, X_{2}}$ is 
integrable, (ii) $\mathbb{E}\left[\left|X_{1}\right|^{2}\right]<\infty$, (iii) $\mathbb{E}\left[X_{2}^{-2}\right]<\infty$, and (iv) $\mathbb{P}\left(X_{2}>0\right)=1$, then

$$
\mathbb{E}\left[R \mathbf{1}_{R<r}\right]=\frac{\varphi_{s 0}(0,0)}{2}-\frac{1}{\pi} \int_{0}^{\infty} \operatorname{Im}\left[\varphi_{s 0}(s,-r s)\right] \frac{\mathrm{d} s}{s},
$$

where

$$
\varphi_{s 0}(s, t) \equiv \int_{-\infty}^{t} \frac{\partial}{\partial s} \varphi_{X_{1}, X_{2}}\left(s, t^{\prime}\right) \mathrm{d} t^{\prime} .
$$

Proof. First, observe that by Hölder's inequality, (ii) and (iii) imply that $\mathbb{E}[|R|]<\infty$. Integrability of the characteristic function ensures that $\left(X_{1}, X_{2}\right)$ has a density, which will be denoted as $f_{X}\left(x_{1}, x_{2}\right)$. Using that $X_{2}$ is almost surely positive,

$$
\begin{aligned}
\mathbb{E}\left[R \mathbf{1}_{R<r}\right] & =\mathbb{E}\left[\frac{X_{1}}{X_{2}} \mathbf{1}_{X_{1}<r X_{2}}\right] \\
& =\mathbb{E}\left[\left(X_{1}-r X_{2}\right) X_{2}^{-1} \mathbf{1}_{X_{1}<r X_{2}}+r \mathbf{1}_{X_{1}<r X_{2}}\right] \\
& =\mathbb{E}\left[\left(X_{1}-r X_{2}\right) X_{2}^{-1} \mathbf{1}_{X_{1}<r X_{2}}\right]+r \mathbb{P}\left(X_{1}<r X_{2}\right) \\
& =\int_{0}^{\infty} \int_{-\infty}^{r x_{2}}\left(x_{1}-r x_{2}\right) x_{2}^{-1} f_{X}\left(x_{1}, x_{2}\right) \mathrm{d} x_{1} \mathrm{~d} x_{2}+r \mathbb{P}\left(X_{1}<r X_{2}\right) .
\end{aligned}
$$

Consider a new random variable $\left(Y_{1}, Y_{2}\right)$ with density

$$
f_{Y}\left(y_{1}, y_{2}\right)=\frac{y_{2}^{-1}}{\mu_{-1}} f_{X}\left(y_{1}, y_{2}\right)
$$

where $\mu_{-1} \equiv \mathbb{E}\left[X_{2}^{-1}\right]$ is finite by assumption. Then

$$
\begin{aligned}
\int_{0}^{\infty} \int_{-\infty}^{r x_{2}}\left(x_{1}-r x_{2}\right) x_{2}^{-1} f_{X}\left(x_{1}, x_{2}\right) \mathrm{d} x_{1} \mathrm{~d} x_{2} & =\mu_{-1} \int_{0}^{\infty} \int_{-\infty}^{r y_{2}}\left(y_{1}-r y_{2}\right) f_{Y}\left(y_{1}, y_{2}\right) \mathrm{d} y_{1} \mathrm{~d} y_{2} \\
& =\mu_{-1} \mathbb{E}\left[W_{r} \mathbf{1}_{W_{r}<0}\right]
\end{aligned}
$$

where $W_{r} \equiv Y_{1}-r Y_{2}$. The characteristic function of $W_{r}$ is

$$
\varphi_{W_{r}}(s)=\varphi_{Y_{1}, Y_{2}}(s,-r s) .
$$

Here, $\varphi_{Y_{1}, Y_{2}}$ denotes the joint characteristic function of $\left(Y_{1}, Y_{2}\right)$, which is

$$
\begin{aligned}
\varphi_{Y_{1}, Y_{2}}(s, t) & =\int_{0}^{\infty} \int_{-\infty}^{\infty} e^{\mathrm{i} s y_{1}+\mathrm{i} t y_{2}} f_{Y}\left(y_{1}, y_{2}\right) \mathrm{d} y_{1} \mathrm{~d} y_{2} \\
& =\int_{0}^{\infty} \int_{-\infty}^{\infty} e^{\mathrm{i} s y_{1}+\mathrm{i} t y_{2}} \frac{y_{2}^{-1}}{\mu_{-1}} f_{X}\left(y_{1}, y_{2}\right) \mathrm{d} y_{1} \mathrm{~d} y_{2} \\
& =\frac{1}{\mu_{-1}} \int_{0}^{\infty} \mathrm{d} y_{1} \int_{-\infty}^{\infty} \mathrm{d} y_{2} f_{X}\left(y_{1}, y_{2}\right)\left[e^{\mathrm{i} s y_{1}+\mathrm{i} l y_{2}} y_{2}^{-1}+\mathrm{i} \int_{l}^{t} e^{\mathrm{i} s y_{1}+\mathrm{i} t^{\prime} y_{2}} \mathrm{~d} t^{\prime}\right] \\
& =\varphi_{Y_{1}, Y_{2}}(s, l)+\frac{\mathrm{i}}{\mu_{-1}} \int_{l}^{t} \varphi_{X_{1}, X_{2}}\left(s, t^{\prime}\right) \mathrm{d} t^{\prime}
\end{aligned}
$$


for any arbitrary but finite $l$. Taking the limit as $l \rightarrow-\infty$,

$$
\varphi_{Y_{1}, Y_{2}}(s, t)=\frac{\mathrm{i}}{\mu_{-1}} \int_{-\infty}^{t} \varphi_{X_{1}, X_{2}}\left(s, t^{\prime}\right) \mathrm{d} t^{\prime}
$$

by the multivariate Riemann-Lebesgue lemma (see, e.g., Stein and Weiss, 1971, Theorem 1.2). Thus,

$$
\varphi_{W_{r}}(s)=\frac{\mathrm{i}}{\mu_{-1}} \int_{-\infty}^{-r s} \varphi_{X_{1}, X_{2}}(s, t) \mathrm{d} t .
$$

Finiteness of $\mathbb{E}\left[X_{1}\right]$ implies that $\varphi_{X_{1}, X_{2}}(s, t)$ is differentiable with respect to $s$, and an application of Leibniz' rule shows the derivative of $\varphi_{W_{r}}(s)$ to be

$$
\begin{aligned}
\varphi_{W_{r}}^{\prime}(s) & \equiv \frac{\mathrm{i}}{\mu_{-1}} \frac{\mathrm{d}}{\mathrm{d} s} \int_{-\infty}^{-r s} \varphi_{X_{1}, X_{2}}(s, t) \mathrm{d} t \\
& =\frac{\mathrm{i}}{\mu_{-1}}\left[-r \varphi_{X_{1}, X_{2}}(s,-r s)+\int_{-\infty}^{-r s} \frac{\partial}{\partial s} \varphi_{X_{1}, X_{2}}(s, t) \mathrm{d} t\right] \\
& =\frac{\mathrm{i}}{\mu_{-1}}\left[-r \varphi_{X_{1}, X_{2}}(s,-r s)+\varphi_{s 0}(s,-r s)\right] .
\end{aligned}
$$

Combining (2.1), (2.2), (2.3) and using Theorem 2.1,

$$
\begin{aligned}
\mathbb{E}\left[R \mathbf{1}_{R<r}\right] & =\mu_{-1} \mathbb{E}\left[W_{r} \mathbf{1}_{W_{r}<0}\right]+r \mathbb{P}\left(X_{1}<r X_{2}\right) \\
& \left.=\mu_{-1}\left[\frac{\varphi_{W_{r}}^{\prime}(0)}{2 \mathrm{i}}+\frac{1}{\pi} \int_{0}^{\infty} \operatorname{Re}\left[\varphi_{W_{r}}^{\prime}(s)\right)\right] \frac{\mathrm{d} s}{s}\right]+r \mathbb{P}\left(X_{1}<r X_{2}\right) \\
& \left.=\frac{\mu_{-1}}{\mathrm{i}} \frac{\varphi_{W_{r}}^{\prime}(0)}{2}+\frac{\mu_{-1}}{\pi} \int_{0}^{\infty} \operatorname{Re}\left[\varphi_{W_{r}}^{\prime}(s)\right)\right] \frac{\mathrm{d} s}{s}+r \mathbb{P}\left(X_{1}<r X_{2}\right) \\
& \left.=-\frac{r}{2}+\frac{\varphi_{s 0}(0,0)}{2}+\frac{\mu_{-1}}{\pi} \int_{0}^{\infty} \operatorname{Re}\left[\varphi_{W_{r}}^{\prime}(s)\right)\right] \frac{\mathrm{d} s}{s}+r \mathbb{P}\left(X_{1}<r X_{2}\right) .
\end{aligned}
$$

Next,

$$
\begin{aligned}
\left.\frac{\mu_{-1}}{\pi} \int_{0}^{\infty} \operatorname{Re}\left[\varphi_{W_{r}}^{\prime}(s)\right)\right] \frac{\mathrm{d} s}{s} & =-\frac{1}{\pi} \int_{0}^{\infty} \operatorname{Im}\left[-r \varphi_{X_{1}, X_{2}}(s,-r s)+\varphi_{s 0}(s,-r s)\right] \frac{\mathrm{d} s}{s} \\
& =\frac{r}{\pi} \int_{0}^{\infty} \operatorname{Im}\left[\varphi_{X_{1}, X_{2}}(s,-r s)\right]-\frac{1}{\pi} \int_{0}^{\infty} \operatorname{Im}\left[\varphi_{s 0}(s,-r s)\right] \frac{\mathrm{d} s}{s} \\
& =\frac{r}{2}-r \mathbb{P}\left(X_{1}<r X_{2}\right)-\frac{1}{\pi} \int_{0}^{\infty} \operatorname{Im}\left[\varphi_{s 0}(s,-r s)\right] \frac{\mathrm{d} s}{s},
\end{aligned}
$$

where the last equality follows from Theorem 2.1 with $n=0$.

We immediately have the following corollary.

Corollary 2.1 Mean of a Ratio. Under the conditions of Theorem 2.2,

$$
\mathbb{E}[R]=\int_{0}^{\infty}\left[\frac{\partial}{\partial s} \varphi_{X_{1}, X_{2}}(s,-t)\right]_{s=0} \mathrm{~d} t
$$

whenever the expectation exists. 
Proof. An argument analogous to the one which led to (2.2) shows that

$$
\mathbb{E}[R]=\mu_{-1} \mathbb{E}\left[W_{r}\right]+r
$$

Set $r=0$ for simplicity. Then, using (2.3),

$$
\begin{aligned}
\mathbb{E}[R] & =\mu_{-1} \mathbb{E}\left[W_{0}\right]=\mu_{-1}(-\mathrm{i}) \varphi_{W_{0}}^{\prime}(0)=\varphi_{s 0}(0,0) \\
& =\int_{-\infty}^{0}\left[\frac{\partial}{\partial s} \varphi_{X_{1}, X_{2}}(s, t)\right]_{s=0} \mathrm{~d} t .
\end{aligned}
$$

A change of variables gives the result.

Corollary 2.1 partially generalizes Lemma 1 of Sawa (1972). Sawa's result applies to higher order moments, but requires the existence of the joint c.f. for purely imaginary arguments, which is not assumed here.

\section{Quadratic Forms in MGH Vectors}

\subsection{Tail Probabilities}

Consider the random variable

$$
L \equiv a_{0}+\mathbf{a}^{\prime} X+X^{\prime} \mathbf{A} X
$$

a quadratic plus a linear form in the random vector $X$. Suppose $X \sim \operatorname{MGHyp}(\boldsymbol{\mu}, \mathbf{C}, \boldsymbol{\gamma}, \lambda, \chi, \psi)$; that is, $X$ has a $d$-variate generalized hyperbolic distribution with stochastic representation

$$
X=\boldsymbol{\mu}+Y \boldsymbol{\gamma}+\sqrt{Y} \mathbf{C} Z
$$

where $Z$ has a $d$-variate standard Normal distribution, $\boldsymbol{\mu}$ and $\boldsymbol{\gamma}$ are constant $d$-vectors, $\mathbf{C}$ is a $d \times d$ matrix, and $Y$ has a univariate generalized inverse Gaussian distribution with density

$$
f_{G I G}(y ; \lambda, \chi, \psi) \equiv \frac{y^{\lambda-1}}{k_{\lambda}(\chi, \psi)} \exp \left\{-\frac{1}{2}\left(\chi y^{-1}+\psi y\right)\right\}
$$

where

$$
k_{\lambda}(\chi, \psi) \equiv\left\{\begin{array}{l}
\frac{\psi}{2}^{-\lambda} \Gamma(\lambda), \text { if } \chi=0 \\
\frac{\chi}{2}^{\lambda} \Gamma(-\lambda), \text { if } \psi=0 \\
2\left(\frac{\chi}{\psi}\right)^{\lambda / 2} K_{\lambda}(\sqrt{\chi \psi}), \text { if } \chi \neq 0 \text { and } \psi \neq 0 .
\end{array}\right.
$$

Here, $K_{\lambda}(z)$ is the modified Bessel function of the second kind of order $\nu$, which, for $\operatorname{Re}(z)>0$, has the integral representation

$$
K_{\lambda}(z) \equiv \frac{1}{2} \int_{0}^{\infty} t^{\lambda-1} \exp \left\{-\frac{1}{2} z\left(t+t^{-1}\right)\right\} \mathrm{d} z
$$

Unlike in the Gaussian case, the characteristic function of $L$ is intractable, so that standard 
results concerning inversion of characteristic functions as used by Imhof (1961) fail. Instead, let $Q \equiv L-a_{0}-\mathbf{a}^{\prime} \boldsymbol{\mu}-\boldsymbol{\mu}^{\prime} \mathbf{A} \boldsymbol{\mu}$ and consider the auxiliary random variable

$$
Q_{0} \equiv \frac{Q}{Y}=\mathbf{a}^{\prime} \boldsymbol{\gamma}+Z^{\prime} \mathbf{C}^{\prime} \mathbf{A} \mathbf{C} Z+2 \boldsymbol{\mu}^{\prime} \mathbf{A} \boldsymbol{\gamma}+\frac{1}{\sqrt{Y}}\left(\mathbf{a}^{\prime} \mathbf{C} Z+2 \boldsymbol{\mu}^{\prime} \mathbf{A C} Z\right)+\sqrt{Y}(2 \boldsymbol{\gamma} \mathbf{A C} Z)+Y \boldsymbol{\gamma}^{\prime} \mathbf{A} \boldsymbol{\gamma}
$$

It will turn out that unlike those of $L$ and $Q$, the joint characteristic function of $Q_{0}$ and $Y^{-1}$, $\varphi_{Q_{0}, Y^{-1}}$ say, is tractable, so that the cdf can be evaluated using the classical inversion formula of Gil-Pelaez (1951), as follows:

$$
\begin{aligned}
\mathbb{P}[Q \leq x] & =\mathbb{P}\left[Q_{0} Y \leq x\right]=\mathbb{P}\left[Q_{0}-x Y^{-1} \leq 0\right] \\
& =\frac{1}{2}-\frac{1}{\pi} \int_{0}^{\infty} \operatorname{Im}\left[\varphi_{Q_{0}, Y^{-1}}(s,-s x)\right] \frac{\mathrm{d} s}{s} .
\end{aligned}
$$

We begin by constructing the spectral decomposition

$$
\mathbf{P} \Lambda \mathbf{P}^{\prime}=\mathbf{C}^{\prime} \mathbf{A C}
$$

where $\boldsymbol{\Lambda}$ is diagonal with entries $\lambda_{j}, j \in\{1, \ldots, d\}$, the eigenvalues of $\mathbf{C}^{\prime} \mathbf{A C}$, and $\mathbf{P}$ is orthogonal. Then $Z^{\prime} \mathbf{C}^{\prime} \mathbf{A C} Z={ }_{d} \sum_{j=1}^{d} \lambda_{j} Z_{j}^{2}$. Further define, for notational convenience, $c \equiv \mathbf{a}^{\prime} \boldsymbol{\gamma}+2 \boldsymbol{\mu}^{\prime} \mathbf{A} \boldsymbol{\gamma}$, $\mathbf{d}=\mathbf{a}^{\prime} \mathbf{C P}+2 \boldsymbol{\mu}^{\prime} \mathbf{A C P}, \mathbf{e}=2 \boldsymbol{\gamma} \mathbf{A C P}$, and $k=\boldsymbol{\gamma}^{\prime} \mathbf{A} \boldsymbol{\gamma}$. Denote by $d_{j}$ and $e_{j}$ the individual elements of $\mathbf{d}$ and $\mathbf{e}$, respectively. We then have the following result.

Theorem 3.1 Distribution of Quadratic Form. Let $L \equiv a_{0}+\mathbf{a}^{\prime} X+X^{\prime} \mathbf{A} X$, where $X \sim$ $\operatorname{MGHyp}(\boldsymbol{\mu}, \mathbf{C}, \boldsymbol{\gamma}, \lambda, \chi, \psi)$. Then

$$
\mathbb{P}[L \leq l]=\frac{1}{2}-\frac{1}{\pi} \int_{0}^{\infty} \operatorname{Im}\left[\Xi_{\lambda}(s,-x s, \chi, \psi)\right] \frac{\mathrm{d} s}{s},
$$

where $x=l-a_{0}-\mathbf{a}^{\prime} \boldsymbol{\mu}-\boldsymbol{\mu}^{\prime} \mathbf{A} \boldsymbol{\mu}$,

$$
\begin{gathered}
\Xi_{\lambda}(s, t, \chi, \psi) \equiv \frac{k_{\lambda}\left(\chi-2 \alpha_{2}(s)-2 \mathrm{i} t, \psi-2 \alpha_{1}(s)\right)}{k_{\lambda}(\chi, \psi)} \rho(s), \\
\alpha_{1}(s) \equiv \mathrm{i} k s-\frac{1}{2} s^{2} \sum_{j=1}^{d} \frac{e_{j}^{2}}{1-2 \mathrm{i} s \lambda_{j}}, \quad \alpha_{2}(s) \equiv-\frac{1}{2} s^{2} \sum_{j=1}^{d} \frac{d_{j}^{2}}{1-2 \mathrm{i} s \lambda_{j}}, \\
\text { and } \rho(s) \equiv \exp \left\{\mathrm{i} s c-s^{2} \sum_{j=1}^{d} \frac{d_{j} e_{j}}{1-2 \mathrm{i} s \lambda_{j}}\right\} \prod_{j=1}^{d} \frac{1}{\sqrt{1-2 \mathrm{i} s \lambda_{j}}} .
\end{gathered}
$$

Proof. Combining (3.1) and (3.2),

$$
\begin{array}{r}
L=a_{0}+\mathbf{a}^{\prime} \boldsymbol{\mu}+\boldsymbol{\mu}^{\prime} \mathbf{A} \boldsymbol{\mu}+\sqrt{Y}\left(\mathbf{a}^{\prime} \mathbf{C} Z+2 \boldsymbol{\mu}^{\prime} \mathbf{A C} Z\right)+Y\left(\mathbf{a}^{\prime} \boldsymbol{\gamma}+Z^{\prime} \mathbf{C}^{\prime} \mathbf{A C} Z+2 \boldsymbol{\mu}^{\prime} \mathbf{A} \boldsymbol{\gamma}\right) \\
+Y^{3 / 2}(2 \boldsymbol{\gamma} \mathbf{A C} Z)+Y^{2} \boldsymbol{\gamma}^{\prime} \mathbf{A} \boldsymbol{\gamma}
\end{array}
$$


Write

$$
Q_{0}={ }_{d} c+k Y+\sum_{j=1}^{d}\left(d_{j} \frac{1}{\sqrt{Y}}+e_{j} \sqrt{Y}\right) Z_{j}+\lambda_{j} Z_{j}^{2} .
$$

The characteristic function of $Q_{0}$, conditional on $Y$, is

$$
\begin{aligned}
\mathbb{E}\left[e^{\mathrm{i} s Q_{0}} \mid Y\right] & =e^{\mathrm{i} s(c+k Y)} \mathbb{E}\left[\exp \left\{\mathrm{i} s \sum_{j=1}^{d}\left(d_{j} \frac{1}{\sqrt{Y}}+e_{j} \sqrt{Y}\right) Z_{j}+\lambda_{j} Z_{j}^{2}\right\}\right] \\
& =e^{\mathrm{i} s(c+k Y)} \prod_{j=1}^{d} \frac{1}{\sqrt{1-2 \mathrm{i} s \lambda_{j}}} \exp \left\{\frac{-s^{2}\left(d_{j} \frac{1}{\sqrt{Y}}+e_{j} \sqrt{Y}\right)^{2}}{2\left(1-2 \mathrm{i} s \lambda_{j}\right)}\right\} \\
& =e^{\mathrm{i} s(c+k Y)} \exp \left\{\frac{-s^{2} \sum_{j=1}^{d}\left(d_{j} \frac{1}{\sqrt{Y}}+e_{j} \sqrt{Y}\right)^{2}}{2\left(1-2 \mathrm{i} s \lambda_{j}\right)}\right\} \prod_{j=1}^{d} \frac{1}{\sqrt{1-2 \mathrm{i} s \lambda_{j}}} \\
& =e^{\alpha_{1}(s) Y+\alpha_{2}(s) Y^{-1}} \rho(s) .
\end{aligned}
$$

We will require the joint characteristic function of $Q_{0}$ and $Y^{-1}$,

$$
\begin{aligned}
\varphi_{Q_{0}, Y^{-1}}(s, t) & \equiv \mathbb{E}\left[e^{\mathrm{i} s Q_{0}+\mathrm{i} t Y^{-1}}\right] \\
& =\mathbb{E}\left[\mathbb{E}\left[e^{\mathrm{i} s Q_{0}+\mathrm{i} t Y^{-1}} \mid Y\right]\right] \\
& =\rho(s) \mathbb{E}\left[e^{\alpha_{1}(s) Y+\left[\alpha_{2}(s)+\mathrm{i} t\right] Y^{-1}}\right] .
\end{aligned}
$$

Using the expression for the density of the generalized inverse Gaussian,

$$
\begin{aligned}
\mathbb{E}\left[e^{\alpha_{1}(s) Y+\left[\alpha_{2}(s)+\mathrm{i} t\right] Y^{-1}}\right] & =\int_{0}^{\infty} e^{\alpha_{1}(s) y+\left[\alpha_{2}(s)+\mathrm{i} t\right] y^{-1}} f_{G I G}(y ; \lambda, \chi, \psi) \mathrm{d} y \\
& =\int_{0}^{\infty} e^{\alpha_{1}(s) y+\left[\alpha_{2}(s)+\mathrm{i} t\right] y^{-1}} \frac{y^{\lambda-1}}{k_{\lambda}(\chi, \psi)} \exp \left\{-\frac{1}{2}\left(\chi y^{-1}+\psi y\right)\right\} \mathrm{d} y \\
& =\int_{0}^{\infty} \frac{y^{\lambda-1}}{k_{\lambda}(\chi, \psi)} \exp \left\{-\frac{1}{2}\left(\left[\chi-2 \alpha_{2}(s)-2 \mathrm{i} t\right] y^{-1}+\left[\psi-2 \alpha_{1}(s)\right] y\right)\right\} \mathrm{d} y \\
& =\frac{k_{\lambda}\left(\chi-2 \alpha_{2}(s)-2 \mathrm{i} t, \psi-2 \alpha_{1}(s)\right)}{k_{\lambda}(\chi, \psi)}
\end{aligned}
$$

so that

$$
\varphi_{Q_{0}, Y^{-1}}(s, t)=\Xi_{\lambda}(s, t, \chi, \psi) \equiv \frac{k_{\lambda}\left(\chi-2 \alpha_{2}(s)-2 \mathrm{i} t, \psi-2 \alpha_{1}(s)\right)}{k_{\lambda}(\chi, \psi)} \rho(s) .
$$

Note that $\operatorname{Re}\left(\alpha_{j}(s)\right)<0, j \in\{1,2\}$, so that together with the integral representation for $K_{\lambda}(z)$ given in (3.3), (3.6) is valid for all $(s, t) \in \mathbb{R}^{2}$.

Using (3.6) in (3.4) and undoing the location shift from $Q$ to $L$ gives the result. 


\subsection{Partial Expectation}

We now turn our attention to the partial expectation of $L$. The challenge in applying Theorem 2.2 is to find an analytic expression for $\varphi_{s 0}(s, t) \equiv \int_{-\infty}^{t} \frac{\partial}{\partial s} \Xi_{\lambda}\left(s, t^{\prime}, \chi, \psi\right) \mathrm{d} t^{\prime}$, where $\Xi_{\lambda}(s, t, \chi, \psi)$ is as in (3.6). Let

$$
\Xi_{\lambda}^{i}(s, t, \chi, \psi) \equiv \frac{k_{\lambda+i}\left(\chi-2 \alpha_{2}(s)-2 \mathrm{i} t, \psi-2 \alpha_{1}(s)\right)}{k_{\lambda}(\chi, \psi)} \rho(s),
$$

so that $\Xi_{\lambda}^{0}(s, t, \chi, \psi) \equiv \Xi_{\lambda}(s, t, \chi, \psi)$. We will need the following result.

LEMMA 3.1. The following relationships hold.

$$
\begin{aligned}
& \text { 1. } \mathrm{i} \int_{-\infty}^{t} \Xi_{\lambda}^{i}\left(s, t^{\prime}, \chi, \psi\right) \mathrm{d} t^{\prime}=\Xi_{\lambda}^{i+1}(s, t, \chi, \psi) . \\
& \text { 2. } \frac{\partial}{\partial t} \Xi_{\lambda}^{i}(s, t, \chi, \psi)=\mathrm{i} \Xi_{\lambda}^{i-1}(s, t, \chi, \psi) . \\
& \text { 3. } \frac{\partial}{\partial s} \Xi_{\lambda}^{i}(s, t, \chi, \psi)=\frac{\mathrm{d} \log \rho(s)}{\mathrm{d} s} \Xi_{\lambda}^{i}(s, t, \chi, \psi)+\frac{\mathrm{d} \alpha_{2}(s)}{\mathrm{d} s} \Xi_{\lambda}^{i-1}(s, t, \chi, \psi)+\frac{\mathrm{d} \alpha_{1}(s)}{\mathrm{d} s} \Xi_{\lambda}^{i+1}(s, t, \chi, \psi) .
\end{aligned}
$$

Proof. See Appendix A.

With this result in hand, the following theorem follows at once.

Theorem 3.2 Partial Expectation of Quadratic Form. Let $L \equiv a_{0}+\mathbf{a}^{\prime} X+X^{\prime} \mathbf{A} X$, $X \sim \operatorname{MGHyp}(\boldsymbol{\mu}, \mathbf{C}, \boldsymbol{\gamma}, \lambda, \chi, \psi)$. Then

$$
\mathbb{E}\left[L \mathbf{1}_{L<l}\right]=(l-x) \mathbb{P}[L<l]+\frac{\varphi_{s 0}(0,0)}{2}-\frac{1}{\pi} \int_{0}^{\infty} \operatorname{Im}\left[\varphi_{s 0}(s,-x s)\right] \frac{\mathrm{d} s}{s},
$$

where $x=l-a_{0}-\mathbf{a}^{\prime} \boldsymbol{\mu}-\boldsymbol{\mu}^{\prime} \mathbf{A} \boldsymbol{\mu}, \varphi_{s 0}(s, t)=\sum_{i=0}^{2} \Xi_{\lambda}^{i}(s, t, \chi, \psi) \beta_{i}(s)$,

$$
\Xi_{\lambda}^{i}(s, t, \chi, \psi) \equiv \frac{k_{\lambda+i}\left(\chi-2 \alpha_{2}(s)-2 \mathrm{i} t, \psi-2 \alpha_{1}(s)\right)}{k_{\lambda}(\chi, \psi)} \rho(s),
$$

$\alpha_{1}(s), \alpha_{2}(s)$, and $\rho(s)$ are as in Theorem 3.1, and $\beta_{0}(s), \beta_{1}(s)$ and $\beta_{2}(s)$ are defined in (3.8-3.10) below.

Proof. Let $x=l-a_{0}-\mathbf{a}^{\prime} \boldsymbol{\mu}-\boldsymbol{\mu}^{\prime} \mathbf{A} \boldsymbol{\mu}$ and $Q=L-a_{0}-\mathbf{a}^{\prime} \boldsymbol{\mu}-\boldsymbol{\mu}^{\prime} \mathbf{A} \boldsymbol{\mu}$ as before. It is easy to see that

$$
\mathbb{E}\left[L \mathbf{1}_{L<l}\right]=\mathbb{E}\left[Q \mathbf{1}_{Q<x}\right]+(l-x) \mathbb{P}[L<l]
$$

Using Theorem 2.2,

$$
\mathbb{E}\left[Q \mathbf{1}_{Q<x}\right]=\frac{\varphi_{s 0}(0,0)}{2}-\frac{1}{\pi} \int_{0}^{\infty} \operatorname{Im}\left[\varphi_{s 0}(s,-x s)\right] \frac{\mathrm{d} s}{s},
$$


where, using Lemma 3.1 twice,

$$
\begin{aligned}
\varphi_{s 0}(s, t) & \equiv \int_{-\infty}^{t} \frac{\partial}{\partial s} \varphi_{Q_{0}, Y^{-1}}\left(s, t^{\prime}\right) \mathrm{d} t^{\prime} \\
& =\frac{\partial}{\partial s} \int_{-\infty}^{t} \Xi_{\lambda}^{i}\left(s, t^{\prime}, \chi, \psi\right) \mathrm{d} t^{\prime} \\
& =\frac{\partial}{\mathrm{i} \partial s} \Xi_{\lambda}^{i+1}(s, t, \chi, \psi) \\
& =\sum_{i=0}^{2} \Xi_{\lambda}^{i}(s, t, \chi, \psi) \beta_{i}(s),
\end{aligned}
$$

and

$$
\begin{gathered}
\beta_{0}(s) \equiv \frac{\mathrm{d} \alpha_{2}(s)}{\mathrm{i} \mathrm{d} s}=\mathrm{i} s \sum_{j=1}^{d} \frac{d_{j}^{2}}{1-2 \mathrm{i} s \lambda_{j}}-s^{2} \sum_{j=1}^{d} \frac{\lambda_{j} d_{j}^{2}}{\left(1-2 \mathrm{i} s \lambda_{j}\right)^{2}}, \\
\beta_{1}(s) \equiv \frac{\mathrm{d} \log \rho(s)}{\mathrm{i} \mathrm{d} s}=c+2 \mathrm{i} s \sum_{j=1}^{d} \frac{d_{j} e_{j}}{1-2 \mathrm{i} s \lambda_{j}}-2 s^{2} \sum_{j=1}^{d} \frac{\lambda_{j} d_{j} e_{j}}{\left(1-2 \mathrm{i} s \lambda_{j}\right)^{2}}+\sum_{j=1}^{d} \frac{\lambda_{j}}{\left(1-2 \mathrm{i} s \lambda_{j}\right)^{2}} \\
\text { and } \beta_{2}(s) \equiv \frac{\mathrm{d} \alpha_{1}(s)}{\mathrm{i} \mathrm{d} s}=k+\mathrm{i} s \sum_{j=1}^{d} \frac{e_{j}^{2}}{1-2 \mathrm{i} s \lambda_{j}}-s^{2} \sum_{j=1}^{d} \frac{\lambda_{j} e_{j}^{2}}{\left(1-2 \mathrm{i} s \lambda_{j}\right)^{2}} .
\end{gathered}
$$

Implementations in Matlab and Fortran of Expressions (3.5) and (3.7) are available from the author upon request. Evaluating them takes typically about a millisecond.

\section{Applications}

\subsection{Sampling Distribution of the 2SLS Estimator}

Consider the simultaneous equations model

$$
\begin{aligned}
& \mathbf{y}_{1}=\mathbf{y}_{2} \beta+\mathbf{X} \boldsymbol{\gamma}+\mathbf{u} \\
& \mathbf{y}_{2}=\mathbf{Z}_{1} \boldsymbol{\pi}+\mathbf{X} \boldsymbol{\delta}+\mathbf{v},
\end{aligned}
$$

where $\mathbf{y}_{1}=\left(y_{1,1}, \ldots, y_{1, n}\right)^{\prime}, \mathbf{y}_{2}=\left(y_{2,1}, \ldots, y_{2, n}\right)^{\prime}, \mathbf{u}=\left(u_{1}, \ldots, u_{n}\right)^{\prime}, \mathbf{v}=\left(v_{1}, \ldots, v_{n}\right)^{\prime}, \mathbf{X}$ is an $n \times k$ matrix of exogenous regressors, $\mathbf{Z}_{1} \notin \mathcal{C}(\mathbf{X})$ is an $n \times k_{1}$ nonstochastic matrix of instruments ${ }^{1}$, and

$$
\left(\begin{array}{l}
u_{i} \\
v_{i}
\end{array}\right) \sim\left(\mathbf{0},\left[\begin{array}{cc}
\sigma_{u}^{2} & \sigma_{u v} \\
\sigma_{u v} & \sigma_{v}^{2}
\end{array}\right]\right),
$$

\footnotetext{
${ }^{1}$ The assumption of nonstochastic instruments is less restrictive than it may appear; if they are indeed stochastic, then it is standard to condition on them, and the analysis proceeds as before.
} 
so that

$$
\varepsilon \equiv\left(\begin{array}{l}
\mathbf{u} \\
\mathbf{v}
\end{array}\right) \sim\left(\mathbf{0},\left[\begin{array}{cc}
\sigma_{u}^{2} \mathbf{I} & \sigma_{u v} \mathbf{I} \\
\sigma_{u v} \mathbf{I} & \sigma_{v}^{2} \mathbf{I}
\end{array}\right]\right) .
$$

Let $\mathbf{M}_{\mathbf{X}} \equiv \mathbf{I}-\mathbf{X}\left(\mathbf{X}^{\prime} \mathbf{X}\right)^{-1} \mathbf{X}^{\prime}$ and define $\mathbf{Z} \equiv \mathbf{M}_{\mathbf{X}} \mathbf{Z}_{1}$. The 2 SLS estimator for $\beta$ is

$$
\hat{\beta}=\frac{\mathbf{y}_{2}^{\prime} \mathbf{P}_{\mathbf{Z}} \mathbf{y}_{1}}{\mathbf{y}_{2}^{\prime} \mathbf{P}_{\mathbf{Z} \mathbf{y}_{2}}}
$$

where $\mathbf{P}_{\mathbf{Z}} \equiv \mathbf{Z}\left(\mathbf{Z}^{\prime} \mathbf{Z}\right)^{-1} \mathbf{Z}^{\prime}$. Let $\hat{B}=\hat{\beta}-\beta$, the estimation error of the 2SLS estimator. Then

$$
\hat{B}=\frac{\boldsymbol{\pi}^{\prime} \mathbf{Z}^{\prime} \mathbf{u}+\mathbf{v}^{\prime} \mathbf{P}_{\mathbf{Z}} \mathbf{u}}{\boldsymbol{\pi}^{\prime} \mathbf{Z}^{\prime} \mathbf{Z} \boldsymbol{\pi}+2 \boldsymbol{\pi}^{\prime} \mathbf{Z}^{\prime} \mathbf{v}+\mathbf{v}^{\prime} \mathbf{P}_{\mathbf{Z} \mathbf{v}}} .
$$

As $\mathbf{P}_{\mathbf{Z}}$ is positive semidefinite, the denominator in (4.3), and hence (4.4), is almost surely positive. Thus

$$
\begin{aligned}
\mathbb{P}[\hat{B} \leq b] & =\mathbb{P}\left[\boldsymbol{\pi}^{\prime} \mathbf{Z}^{\prime} \mathbf{u}+\mathbf{v}^{\prime} \mathbf{P}_{\mathbf{Z}} \mathbf{u}-b\left(\boldsymbol{\pi}^{\prime} \mathbf{Z}^{\prime} \mathbf{Z} \boldsymbol{\pi}+2 \boldsymbol{\pi}^{\prime} \mathbf{Z}^{\prime} \mathbf{v}+\mathbf{v}^{\prime} \mathbf{P}_{\mathbf{Z}} \mathbf{v}\right) \leq 0\right] \\
& =\mathbb{P}\left[a_{0}+\mathbf{a}^{\prime} \boldsymbol{\varepsilon}+\boldsymbol{\varepsilon}^{\prime} \mathbf{A} \boldsymbol{\varepsilon} \leq 0\right]
\end{aligned}
$$

where

$$
a_{0} \equiv-b \boldsymbol{\pi}^{\prime} \mathbf{Z}^{\prime} \mathbf{Z} \boldsymbol{\pi}, \quad \mathbf{a} \equiv\left(\begin{array}{c}
\mathbf{Z} \boldsymbol{\pi} \\
-2 b \mathbf{Z} \boldsymbol{\pi}
\end{array}\right), \quad \text { and } \mathbf{A} \equiv \frac{1}{2}\left(\begin{array}{cc}
\mathbf{0} & \mathbf{P}_{\mathbf{Z}} \\
\mathbf{P}_{\mathbf{Z}} & -2 b \mathbf{P}_{\mathbf{Z}}
\end{array}\right) .
$$

Equation (4.5) is in the required form, so that Theorem 3.1 is readily applied. Hence, the exact sampling distribution of the 2SLS estimator can be evaluated for an arbitrary but known (or consistently estimated) covariance structure, and under MGHyp errors.

\subsection{VaR and ES of a Quadratic Portfolio}

Consider a probability space $(\Omega, \mathcal{F}, \mathbb{P})$. The Expected Shortfall at level $\alpha \in(0,1)$ of a position with terminal value $X: \Omega \rightarrow \mathbb{R}$ is defined as (Acerbi and Tasche, 2002a,b)

$$
E S^{(\alpha)} \equiv-\frac{1}{\alpha}\left[\mathbb{E}\left[X \mathbf{1}_{X \leq x^{(\alpha)}}\right]-x^{(\alpha)}\left(\mathbb{P}\left[X \leq x^{(\alpha)}\right]-\alpha\right)\right]
$$

where

$$
x^{(\alpha)} \equiv \inf \{x: \mathbb{P}[X \leq x] \geq \alpha\}
$$

is the $\alpha \times 100 \%$ quantile of the distribution of $X$. Often $\alpha=1 \%$. Apart from the sign, $x^{(\alpha)}$ corresponds to the Value at Risk (VaR) at level $\alpha$, which is a risk measure in its own right.

The definition in (4.6) ensures that $E S^{(\alpha)}$ is a coherent risk measure in the sense of Artzner et al. (1999) even if $X$ is not absolutely continuous. In our setting, $X$ is indeed absolutely continuous, so that the Expected Shortfall, expressed in terms of the loss $L=-X$, reduces to

$$
E S_{L}^{(\alpha)} \equiv \frac{1}{\alpha}\left[\mathbb{E}\left[L \mathbf{1}_{L>l^{(1-\alpha)}}\right]\right]
$$


where

$$
\mathbb{P}\left[L>l^{(1-\alpha)}\right]=\alpha .
$$

As in Broda (2011), consider a portfolio with associated loss $L=-X$, depending on $d$ risk factors $S=\left(S_{1}, \ldots, S_{d}\right)^{\prime}$. For a given time horizon $\tau$, the portfolio loss is modeled as a quadratic function of $\Delta S \equiv S-S_{0}$, the changes in the risk factors. That is,

$$
L=a_{0}+\mathbf{a}^{\prime} \Delta S+\Delta S^{\prime} \mathbf{A} \Delta S,
$$

for some scalar constant $a_{0}$ and, respectively, a conformable vector a and matrix $\mathbf{A}$. Because $\Delta S^{\prime} \mathbf{A} \Delta$ is a scalar, we can take $\mathbf{A}$ to be symmetric without loss of generality. A quadratic portfolio of this type typically arises from a Delta-Gamma-Theta approximation, which is quite popular among risk managers. In it, one takes $a_{0}=-\tau \Theta, \mathbf{a}=-\boldsymbol{\delta}$, and $\mathbf{A}=-\frac{1}{2} \boldsymbol{\Gamma}$. Here $\delta_{j}=\partial X / \partial S_{j}, \Gamma_{j k}=\partial^{2} X / \partial S_{j} \partial S_{k}$, and $\Theta=-\partial X / \partial \tau$ are the so-called "Greeks".

\section{Conclusions}

Quadratic forms are ubiquitous in Econometrics: according to Google Scholar, the paper of Imhof (1961) — which provides an algorithm for evaluating their distribution function under Gaussianity - has 931 citations. The results herein generalize Imhof's result to the MGhyp distribution, thus allowing for skewness and heavy tails, which is critically important in finance in particular. The expressions provided in the paper can be quickly and reliably evaluated in a computer, with code available from the author, making them easy to use in applied work.

There are a number of aspects that the author would like to explore in subsequent work. The first question is whether as in Broda (2011), conditions can be established under which the integrals in Theorems 3.1 and 3.2 can be expanded in a uniform asymptotic expansion, hence removing the need for numerical integration. Relatedly, under the same conditions, it should be possible to derive an importance sampling algorithm as in Glasserman et al. (2002). This is particularly useful in the portfolio risk application of Section 4.2 above, in cases where the quadratic approximation to the portfolio value is poor. Finally, concerning the 2SLS application in Section 4.1, it is of interest to analyze the joint distribution of the structural estimates in a model with two endogenous regressors.

\section{References}

Abramowitz, M. And I. Stegun, eds. (1964): Handbook of Mathematical Functions with Formulas, Graphs, and Mathematical Tables, New York: Dover. 17

Acerbi, C. And D. Tasche (2002a): "Expected Shortfall: A Natural Coherent Alternative to Value at Risk," Economic Notes, 31, 379-388. 12

(2002b): "On the Coherence of Expected Shortfall," Journal of Banking and Finance, 26, 1487-1503. 12 
Anderson, T. W. And T. Sawa (1973): "Distributions of Estimates of Coefficients of a Single Equation in a Simultaneous System and their Asymptotic Expansions," Econometrica, 41, $683-714.1$

Artzner, P., F. Delbaen, J.-M. Eber, and D. Heath (1999): "Coherent Measures of Risk," Mathematical Finance, 9, 203-228. 2, 12

Barndorff-Nielsen, O. E. (1977): "Exponentially Decreasing Distributions for the Logarithm of Particle Size," Proceedings of the Royal Society of London, Series A, 353, 401-419. 1

Blæsild, P. And J. L. Jensen (1981): "Multivariate Distributions of Hyperbolic Type," in Statistical Distributions in Scientific Work, ed. by C. Taillie, G. Patil, and B. Baldessari, Dordrecht: Reidel, vol. 4, 45-66. 1

Bormetti, G., V. Cazzola, G. Livan, G. Montagna, and O. Nicrosini (2010): "A Generalized Fourier Transform Approach to Risk Measures," Journal of Statistical Mechanics: Theory and Experiment, 2010, P01005. 2

Broda, S. A. (2011): "The Expected Shortfall of Quadratic Portfolios with Heavy-Tailed Risk Factors," Mathematical Finance, forthcoming. 2, 13

Broda, S. A. And M. S. Paolella (2009): "CHICAGO: A Fast and Accurate Method for Portfolio Risk Calculation," Journal of Financial Econometrics, 7, 412-436. 2

Butler, R. W. And A. T. A. Wood (2004): "Saddlepoint Approximation for Moment Generating Functions of Truncated Random Variables," Annals of Statistics, 32, 2712-2730. 2

Cribbett, P. F., J. N. Lye, And A. Ullah (1989): "Evaluation of the Two-Stage Least Squares Distribution Function by Imhof's Procedure," Journal of Quantitative Economics, 5, 91-96. 2

Dickey, D. A. And W. A. Fuller (1979): "Distribution of the Estimators for Autoregressive Time Series With a Unit Root," Journal of the American Statistical Association, 74, pp. 427431. 1

Durbin, J. And G. S. Watson (1950): "Testing for Serial Correlation in Least Squares Regression, I." Biometrika, 37, 409-428. 1

Eberlein, E. And U. Keller (1995): "Hyperbolc Distributions in Finance," Bernoulli, 1, 281-299. 1

Forchini, G. (2002): "The Exact Cumulative Distribution Function of a Ratio of Quadratic Forms in Normal Variables, with Application to the AR(1) Model," Econometric Theory, 18, 823-852. 1

(2006): "On the Bimodality of the Exact Distribution of the TSLS Estimator," Econometric Theory, 22, 932-946. 1 
Gil-Pelaez, J. (1951): "Note on the Inversion Theorem," Biometrika, 38, 481-82. 1, 2, 3, 4, 8

Glasserman, P., P. Heidelberger, and P. Shahabuddin (2002): "Portfolio Value-at-Risk with Heavy-Tailed Risk Factors," Mathematical Finance, 12, 239-269. 1, 13

Hellmich, M. and S. Kassberger (2011): "Efficient and Robust Portfolio Optimization in the Multivariate Generalized Hyperbolic Framework," Quantitative Finance, 11, 1503-1516. 1

Hillier, G. (2006): "Yet more on the Exact Properties of IV Estimators," Econometric Theory, $22,913-931.1$

Holly, A. And P. C. B. Phillips (1979): "A Saddlepoint Approximation to the Distribution of the $k$-Class Estimator of a Coefficient in a Simultaneous System," Econometrica, 47, 1527-1547. 1

Imнof, J. P. (1961): "Computing the Distribution of Quadratic Forms in Normal Variables," Biometrika, 48, 419-26. 1, 8, 13

Kim, Y. S., S. T. Rachev, M. L. Bianchi, and F. J. Fabozzi (2009): "Computing VaR and AVaR In Infinitely Divisible Distributions," Technical report, University of Karlsruhe. 2

KNight, J. L. (1986): "Non-Normal Errors and the Distribution of OLS and 2SLS Structural Estimators," Econometric Theory, 2, 75-106. 1

Kwiatkowski, D., P. C. B. Phillips, P. Schmidt, and Y. Shin (1992): "Testing the Null Hypothesis of Stationarity Against the Alternative of a Unit Root," Journal of Econometrics, $54,91-115.1$

Lukacs, E. (1970): Characteristic Functions, London: Griffin, 2nd ed. 3

Maddala, G. S. and J. Jeong (1992): "On the Exact Small Sample Distribution of the Instrumental Variable Estimator," Econometrica, 60, 181-183. 1

Martin, R. (2006): "The Saddlepoint Method and Portfolio Optionalities," Risk Magazine, 19, 93-95. 2

Nelson, C. R. and R. Startz (1990a): "The Distribution of the Instrumental Variables Estimator and Its t-Ratio When the Instrument is a Poor One," The Journal of Business, 63 (Part 2), S125-S140. 1

(1990b): "Some Further Results on the Exact Small Sample Properties of the Instrumental Variable Estimator," Econometrica, 58, 967-976. 1

Ooura, T. And M. Mori (1991): "The Double Exponential Formula for Oscillatory Functions Over the Half Infinite Interval," Journal of Computational and Applied Mathematics, 38, 353360. 4 
Phillips, P. C. B. (2006): "A Remark on Bimodality and Weak Instrumentation in Structural Equation Estimation," Econometric Theory, 22, 947-960. 1

Pinelis, I. (2010): "Positive-Part Moments via the FourierLaplace Transform," Journal of Theoretical Probability. 2

Richardson, D. H. (1968): "The Exact Distribution of a Structural Coefficient Estimator," Journal of the American Statistical Association, 63, 1214-1225. 1

Rosén, B. (1961): "On the Asymptotic Distribution of Sums of Independent Identically Distributed Random Variables," Arkiv för Matematik, 4, 323-332. 4

SAwA, T. (1969): "The Exact Sampling Distributions of Ordinary Least Squares and Two Stage Least Squares Estimates," Journal of the American Statistical Association, 64, 923-980. 1

(1972): "Finite-Sample Properties of the $k$-Class Estimators," Econometrica, 40, 653-680. 7

Stein, E. M. And G. Weiss (1971): Introduction to Fourier Analysis on Euclidean Spaces, Princeton: Princeton University Press. 6

Wendel, J. G. (1961): “The Nonabsolute Convergence of Gil-Pelaez' Inversion Integral," Annals of Mathematical Statistics, 32, 338-339. 4

Woglom, G. (2001): "More Results on the Exact Small Sample Properties of the Instrumental Variable Estimator," Econometrica, 69, 1381-1389. 1

Yueh, M.-L. And M. C. W. Wong (2010): "Analytical VaR and Expected Shortfall for Quadratic Portfolios," Journal of Derivatives, 17, 33-44. 2

\section{A Proofs}

Proof of Lemma 3.1. The result is trivial if $\chi=0$ or $\psi=0$, so we only consider the case in which both $\chi$ and $\psi$ are nonzero.

1.: We have that

$$
\begin{aligned}
\mathrm{i} \int_{-\infty}^{t} \Xi_{\lambda}^{i}\left(s, t^{\prime}, \chi, \psi\right) \mathrm{d} t^{\prime} & =\mathrm{i} \int_{-\infty}^{t} \frac{k_{\lambda+i}\left(\chi-2 \alpha_{2}(s)-2 \mathrm{i} t^{\prime}, \psi-2 \alpha_{1}(s)\right)}{k_{\lambda}(\chi, \psi)} \rho(s) \mathrm{d} t^{\prime} \\
& =\frac{2 \mathrm{i} \rho(s)}{k_{\lambda}(\chi, \psi)} \int_{-\infty}^{t}\left(\frac{u\left(t^{\prime}\right)}{\psi-2 \alpha_{1}(s)}\right)^{\lambda+i} K_{\lambda+i}\left(u\left(t^{\prime}\right)\right) \mathrm{d} t^{\prime}
\end{aligned}
$$

where $u(t) \equiv \sqrt{\left(\chi-2 \alpha_{2}(s)-2 \mathrm{i} t\right)\left(\psi-2 \alpha_{1}(s)\right)}$, so that $\mathrm{d} t^{\prime}=\mathrm{i} u\left(\psi-2 \alpha_{1}(s)\right)^{-1} \mathrm{~d} u$. Changing 
variables,

$$
\begin{aligned}
\frac{2 \mathrm{i} \rho(s)}{k_{\lambda}(\chi, \psi)} \int_{-\infty}^{t}\left(\frac{u\left(t^{\prime}\right)}{\psi-2 \alpha_{1}(s)}\right)^{\lambda+i} K_{\lambda+i}\left(u\left(t^{\prime}\right)\right) \mathrm{d} t^{\prime} & =\frac{-2 \rho(s)}{k_{\lambda}(\chi, \psi)\left(\psi-2 \alpha_{1}(s)\right)^{\lambda+i+1}} \int_{-\infty}^{u(t)} u^{\lambda+i+1} K_{\lambda+i}(u) \mathrm{d} u \\
& =\frac{2 \rho(s)}{k_{\lambda}(\chi, \psi)}\left(\frac{u(t)}{\psi-2 \alpha_{1}(s)}\right)^{\lambda+i+1} K_{\lambda+i+1}(u(t)) \Leftrightarrow \\
\mathrm{i} \int_{-\infty}^{t} \Xi_{\lambda}^{i}\left(s, t^{\prime}, \chi, \psi\right) \mathrm{d} t^{\prime} & =\Xi_{\lambda}^{i+1}(s, t, \chi, \psi),
\end{aligned}
$$

where the penultimate equality follows from Abramowitz and Stegun (1964, Eq. 11.3.18).

2.: Differentiating (A.1) immediately yields

$$
\frac{\partial}{\partial t} \Xi_{\lambda}^{i}(s, t, \chi, \psi)=\mathrm{i} \Xi_{\lambda}^{i-1}(s, t, \chi, \psi) .
$$

3.: First observe that

$$
\begin{aligned}
\frac{\partial}{\partial \psi} k_{\lambda}(\chi, \psi) & =2 \frac{\partial}{\partial \psi}\left(\frac{\chi}{\psi}\right)^{\lambda / 2} K_{\lambda}(\sqrt{\chi \psi}) \\
& =2\left[\frac{1}{2 \psi}\left(\frac{\chi}{\psi}\right)^{\lambda / 2} K_{\lambda}^{\prime}(\sqrt{\chi \psi}) \sqrt{\chi \psi}-\frac{\lambda}{2 \psi}\left(\frac{\chi}{\psi}\right)^{\lambda / 2} K_{\lambda}(\sqrt{\chi \psi})\right] \\
& =\left(\frac{\chi}{\psi}\right)^{\lambda / 2} \frac{1}{\psi}\left[K_{\lambda}^{\prime}(\sqrt{\chi \psi}) \sqrt{\chi \psi}-\lambda K_{\lambda}(\sqrt{\chi \psi})\right] \\
& =\left(\frac{\chi}{\psi}\right)^{\lambda / 2} \frac{1}{\psi}\left[-\sqrt{\chi \psi} K_{\lambda+1}(\sqrt{\chi \psi})\right]=-\frac{1}{2} k_{\lambda+1}(\chi, \psi)
\end{aligned}
$$

where the penultimate equality follows from Abramowitz and Stegun (1964, Eq. 9.6.28). Then, indulging in some abuse of notation,

$$
\begin{aligned}
& \frac{\partial}{\partial s} \Xi_{\lambda}^{i}(s, t, \chi, \psi)= \frac{\partial}{\partial s} \frac{k_{\lambda+i}\left(\chi-2 \alpha_{2}(s)-2 \mathrm{i} t, \psi-2 \alpha_{1}(s)\right)}{k_{\lambda}(\chi, \psi)} e^{\log (\rho(s))} \\
&= \frac{\mathrm{d} \log \rho(s)}{\mathrm{d} s} \Xi_{\lambda}^{i}(s, t, \chi, \psi)+\frac{\mathrm{d} \alpha_{2}(s)}{\mathrm{d} s} \frac{\partial}{\mathrm{i} \partial t} \Xi_{\lambda}^{i}(s, t, \chi, \psi) \\
& \quad-\frac{2 \rho(s)}{k_{\lambda}(\chi, \psi)} \frac{\mathrm{d} \alpha_{1}(s)}{\mathrm{d} s} \frac{\partial}{\partial \psi} k_{\lambda+i}\left(\chi-2 \alpha_{2}(s)-2 \mathrm{i} t, \psi-2 \alpha_{1}(s)\right) \\
&= \frac{\mathrm{d} \log \rho(s)}{\mathrm{d} s} \Xi_{\lambda}^{i}(s, t, \chi, \psi)+\frac{\mathrm{d} \alpha_{2}(s)}{\mathrm{d} s} \Xi_{\lambda}^{i-1}(s, t, \chi, \psi) \\
& \quad+\frac{\rho(s)}{k_{\lambda}(\chi, \psi)} \frac{\mathrm{d} \alpha_{1}(s)}{\mathrm{d} s} k_{\lambda+i+1}\left(\chi-2 \alpha_{2}(s)-2 \mathrm{i} t, \psi-2 \alpha_{1}(s)\right) \\
&=\frac{\mathrm{d} \log \rho(s)}{\mathrm{d} s} \Xi_{\lambda}^{i}(s, t, \chi, \psi)+\frac{\mathrm{d} \alpha_{2}(s)}{\mathrm{d} s} \Xi_{\lambda}^{i-1}(s, t, \chi, \psi)+\frac{\mathrm{d} \alpha_{1}(s)}{\mathrm{d} s} \Xi_{\lambda}^{i+1}(s, t, \chi, \psi),
\end{aligned}
$$

where the final equality follows by using (A.2) and (A.3). 